\title{
Error-correcting Coding Usage for Data Transmission through Power Lines
}

\author{
A. V. Ishchenko', V. S. Shevelev' ${ }^{2}$ A. S. Naronov $^{3}$, A. N. Tcherepanov ${ }^{4}$ B. V. Shulgin ${ }^{5}$ \\ $1,2,4,5$ Department of Experimental Physics, Ural Federal University, Russia \\ ${ }^{3}$ Scientific and Production Association of Automatics Named after Academician N.A. Semikhatov, Russia
}

\begin{abstract}
Article Info
Article history:

Received Nov 29, 2017

Revised Jul 1, 2018

Accepted Aug 6, 2018

\section{Keyword:}

Communication line Downhole telemetry systems

ABSTRACT

A concept of data transmission within downhole telemetry systems in oilfield industry through power lines is presented. Based on this concept, MATLAB/Simulink models simulating communication lines in downhole telemetry systems are built, which can be used as prototypes for development of real systems. The most appropriate signal modulation methods for data transmission in downhole telemetry systems are suggested and discussed. The influence of high-voltage interference on signal transmission through power line from downhole unit to ground based unit is simulated. Usage of error-correcting coding methods for data transmission such as Hamming code, Reed-Solomon code, BCH code is suggested, and its efficiency is demonstrated.
\end{abstract} Error-correcting coding Power line
Copyright $(0) 2018$ Institute of Advanced Engineering and Science. All rights reserved.

\section{Corresponding Author:}

Aleksey Ishchenko,

Departement of Experimenal Physics,

Ural Federal University,

Mira 19, Yekaterinburg, 620002, Russia

Email: a-v-i@mail.ru

\section{INTRODUCTION}

Downhole telemetry systems (DHTS) are widely used in oilfield industry for in-well monitoring. In this field, many problems still remain unsolved. Standard circuit solutions and electronic components do not allow to provide high-quality communications between Data Acquisition Equipment (ground based unit GBU) located at the surface and submersible units (downhole unit - DU), which complicates controlling over a downhole equipment operation mode and makes oil production less efficient and safe. For development of modern DHTSs meeting the above mentioned requirements an information transmission method should be selected in the first place. Today, the power line communication technique seems more promising method for DHTSs. Such technique enables to carry communication signals through power conductors. Generally, the motivation is to use existing power supply infrastructures [1,2]. There are various ways to transmit information in power lines. For example, information can be transmitted by phase-to-phase communication scheme [2]. For high-bitrate communication in some cases a multi-conductor cable can be used [3, 4].

Within this area of focus, we have performed a series of works aimed at development of downhole telemetry systems. A method for protection of a downhole unit (DU) against high voltage interferences in data transmission lines has been suggested [5]. DU reliability calculations for severe operation conditions have been made [6]. Equivalent circuit diagrams for substitution of a powerline used for data transmission have been calculated [7], and a concept of a test stand simulating a downhole telemetry system has been presented [8]. In this work, results of performed simulation of data transmission through a power line by error-correcting coding methods are presented. Furthermore, signal modulation and demodulation simulation results are shown, and modulated signal transmission through a communication line under conditions of high voltage interferences and noise is discussed. 


\section{STRUCTURE DIAGRAM OF A DATA TRANSMISSION CHANNEL}

For data transmission within DHTS from DU to GBU a structure diagram of a downhole telemetry system has been developed as shown in Figure 1. We have simulated the substitution of a communication line, and the simulation results are shown in [7].

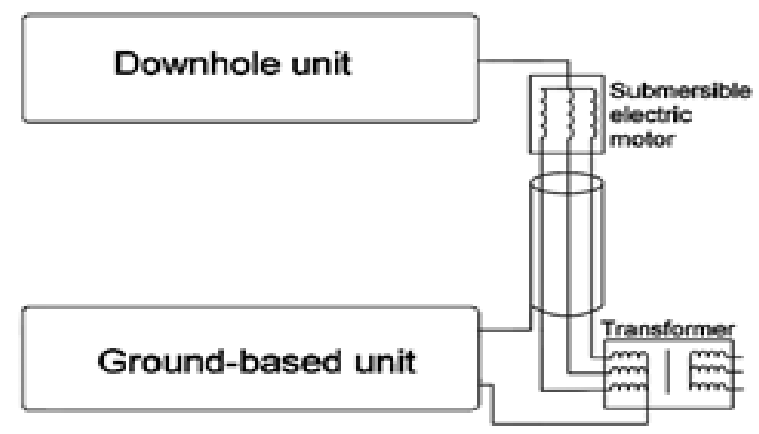

Figure 1. Structure diagram of a downhole telemetry system

DHTS consists of 2 main system units and a power transmission cable intended for power supply to a submersible electromotor (SEM). This cable will serve as a physical medium for data transmission from the DU to the GBU.

It was found formerly [7] that the data transmission process in such systems carries in a long distance communication line in the context of intensive interferences; whereas requirements to reliability and accuracy of data transmitted remain high. In this regard, a structure diagram is suggested for transmission of the signal informative element to DHTS (Figure 2). Figure 2 shows the main components of the system: modulator/demodulator and encoder/decoder. The modulator and demodulator converse an information signal into the physical one and back, respectively. Binary frequency-shift keying (BFSK) and binary phaseshift keying (BPSK) modulation methods are selected as the most appropriate signal modulation methods.

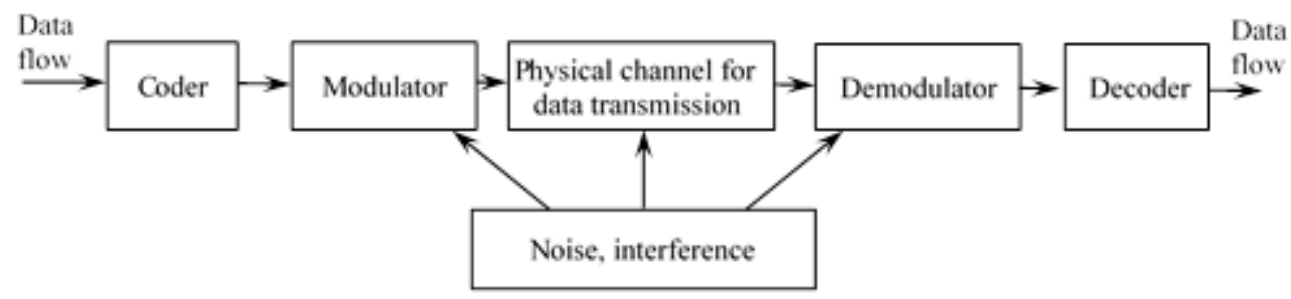

Figure 2. Structure diagram of DHTS information element by data transmission through power transmission cable

In this case the encoder and decoder represent a software implementation of mathematical algorithms for error-correcting data coding and decoding. Interferences and noises shown as a separate block on the structure diagram (Figure 2) can affect not only signals to be transmitted in a communication line, but also signal modulation and demodulation units. In addition, SEM power supply interrupts and voltage peaks in SEM power supply line often occur during data transmission through DHTS communication cables. These interrupts and peaks result in loss of not only single bits, but also entire data blocks. Therefore, data transmission in DHTS requires, at our opinion, application of sophisticated combined coding systems based on correlative and iterative coding and on different types of $\mathrm{BCH}$ codes (Bose-Chaudhuri-Hocquenghem codes). However, application of codes with sophisticated processes for signal coding is likely to be impracticable because DU has a limited computing capability. On the other hand, complexity of information decoding imposes no significant restrictions, since decoding is performed at the receiver side (GBU), where no limitations in usage of highly capable computing devices exist. With this background, we have surveyed codes for error-correcting coding that show good results in coding of audio and video content flows, space communications, fax communications and in many other applications. Upon results of this survey, two types 
of data coding methods have been selected: $\mathrm{BCH}$ codes and their special case, Reed-Solomon codes. These codes are used the most frequently when multiple errors and data block losses are possible. The coding simplicity of these codes is their apparent advantage due to the fat that it implies significant relaxation in requirements for performance rates of high-temperature microcontrollers included in DU and working in the well.

\section{SIMULATION MODEL OF DATA TRANSMISSION IN POWER CABLES}

According to the above mentioned concept, a model simulating a communication line for telemetry data transmission in a power cable is developed. Results of works described formerly in [7] are used for the communication line model. The model is implemented in MATLAB/Simulink environment and shown on Figures 3 and 4. MATLAB/Simulink simulation environment is chosen for solution of the set tasks due to the fact that it enables to use ready block libraries for simulation of power supply, mechanical and hydraulic systems, as well as to employ an advanced model-oriented approach to design of control systems, digital communication techniques and real-time devices. Simulink environment has also shown good results in simulation of various electrical motors and devices for power supply electronics [9-11].

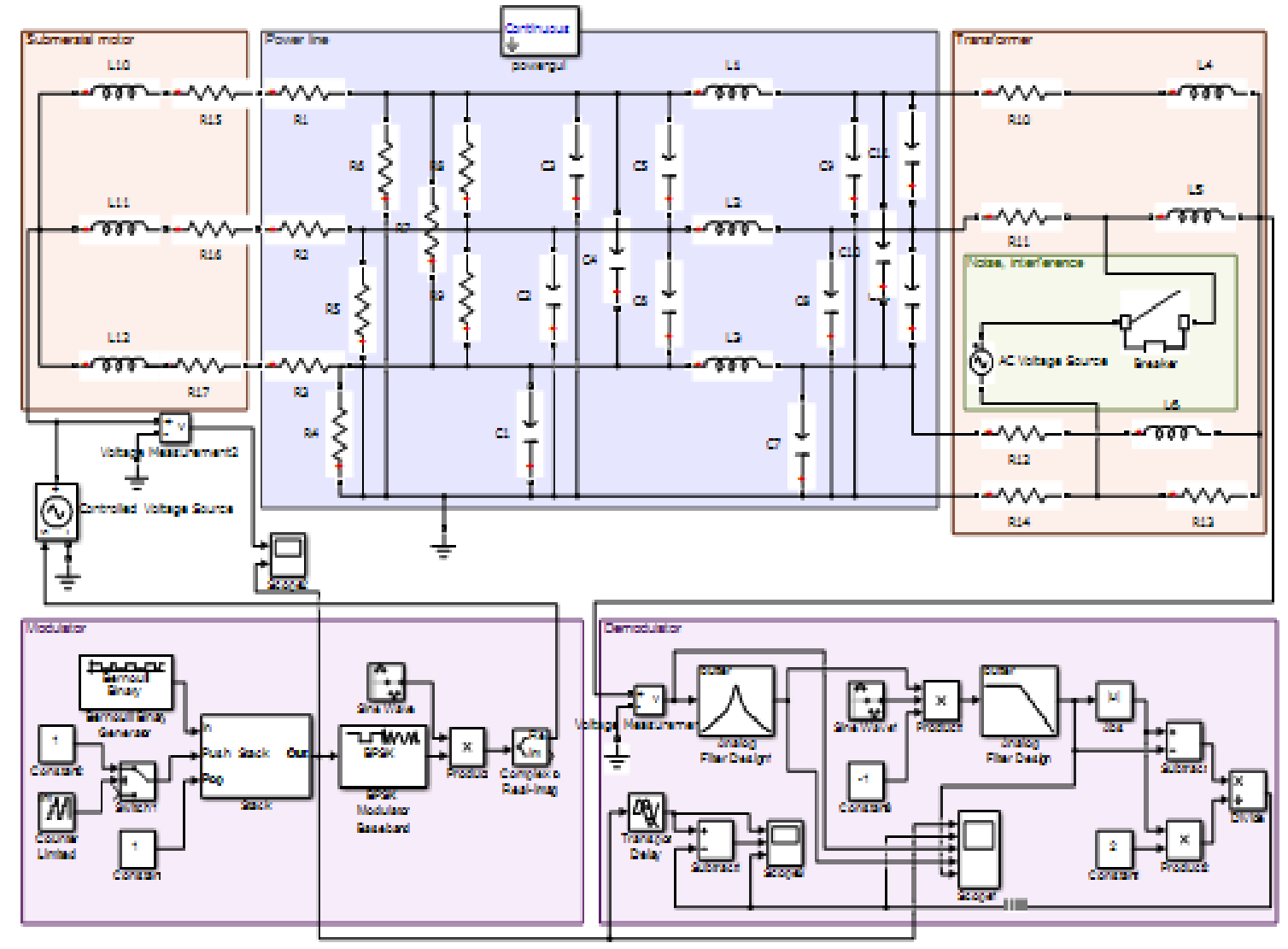

Figure 3. Communication line simulation model with regard to the signal transmission physical model

The presented model can be provisionally divided into two parts. The first part is responsible for transmission of physical signals in the communication line Figure 3). The model considers a representation of the bit data modulation and demodulation process and enables to describe system's behavior under the influence of the high-voltage interference. The second part describes the process of signal informative element transmission in the communication line (Figure 4). In addition to the modulator and demodulator, this part includes also such blocks as the encoder and decoder with different types of data coding methods. This part does not include blocks containing model representations of the signal physical element to be transmitted in the communication line. However, specific details of data transmission in a real 
communication line are considered on simulating of the bit flow transmission process in an additive white Gaussian noise channel (AWGN channel).

The model on Figure 3 includes following main blocks: the modulator with the binary signal source, the physical model of the communication line, the signal demodulator. The binary signal source, in this case, is the generator of random binary single-digit numbers, probability of which follows the Bernoulli distribution. In the said model experiments the probability of 0 or 1 is set as equal to 0.5 .

The signal modulator represents a complex of blocks functionally implementing a BSPK modulation method. The modulator (Figure 3) includes the Sine Wave block generating sine harmonics used as a carrier for phase modulation. The carrier signal frequency is selected as $6 \mathrm{kHz}$. Binary data are created by the binary number generator with Bernoulli distribution (Bernoulli Binary Generator) using a frequency demultiplier built on Stack, Switch, Counter Limited blocks and Constant1 and Constant 2 blocks. The random bit stream rate is determined by the elapsed time till the counter overflow. When the counter overflow signal is generated, Switch1 transfers the signal "1" to the control input of the stack register PUSH. At that moment the random data from the Bernoulli binary generator are recorded into the stack register and stored there till the entry of the next permission signal into the PUSH input. Thereby, the created generator enables to simulate a stream of random binary data with different baud rates. To simulate operation, the random number generator is set to generation of data with data size of 1024 bits with the transfer rate of 300 bits per second. It should be noted that the most difficult case of bit-stream transferring in view of data reliability is the transfer of a bit stream with random data. If the bit stream is divided into packages of a strictly determined structure, data receiving can be performed with a higher reliability; that's why random signals will be used for simulation.

The generated binary data flow comes to the input of the modulator block (BPSK Modulator Baseband). Further, the carrier signal is modulated using "Product" block. Signal conversion from Simulink mathematical model into the physical form is performed using a controlled voltage source. Use of BPSK modulation method that is simpler in comparison with QPSK and 8-PSK methods s caused by lower requirements for data transmission channels, in particular, for SNR level of the transmission line. Thus, as shown in [12], data transmission using BPSK method is the most noise-proof, if all other parameters are equal.

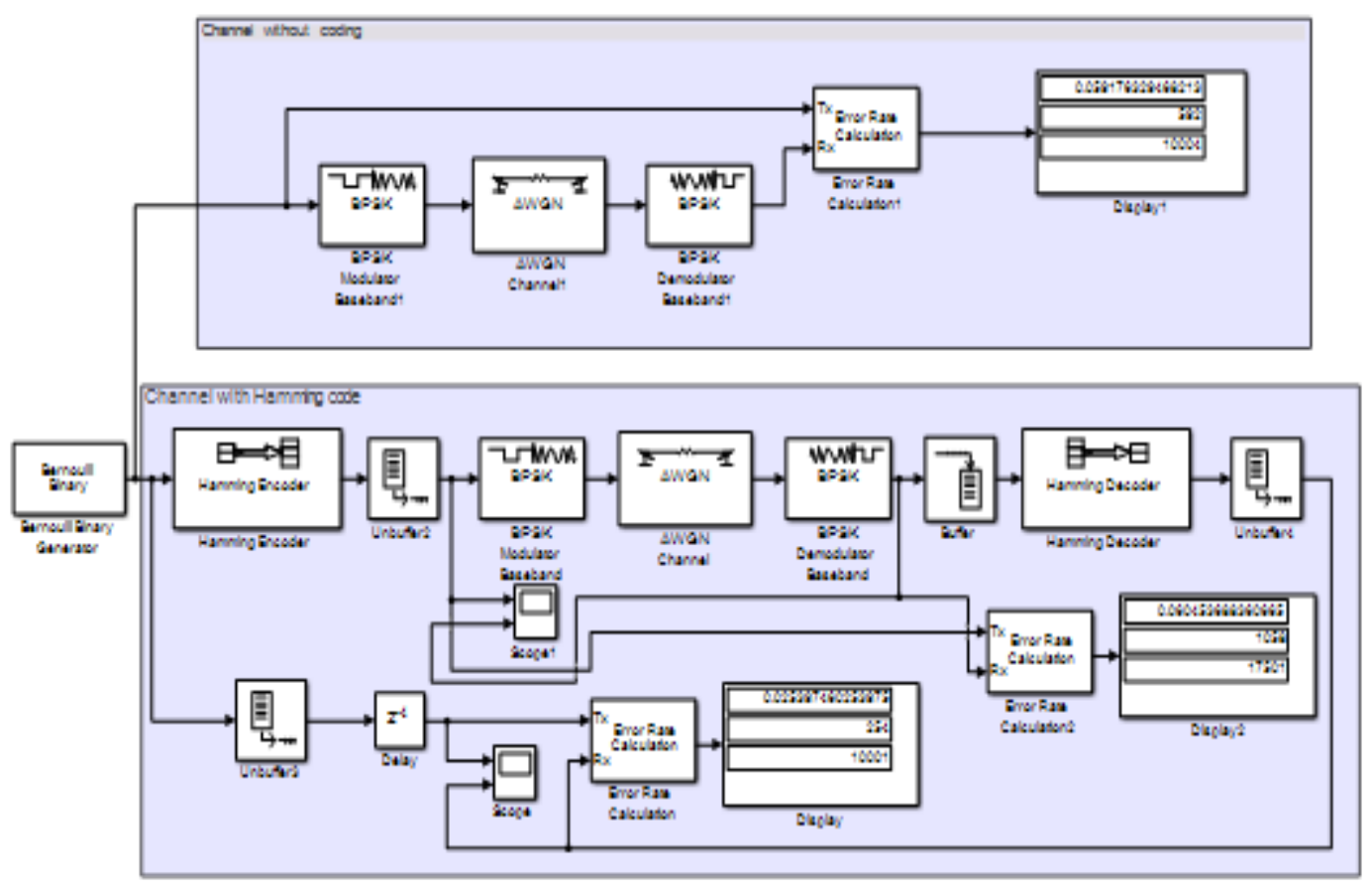

Figure 4. Bit data transmission model in communication lines using Hamming code

The signal demodulator used in this model is furnished according to a correlative scheme (Figure 3). This scheme is characterized with a relatively easiness of implementation and sufficient detection quality 
level for signals with BPSK and BFSK modulations. The base unit of this scheme is the correlator based on a multiplier and integrator. The demodulator (Figure 3) has following functions. The incoming signal received from the communication line is conversed from a physical form into the Simulink signal through Voltage Measurement 1 convertor enters the input of a narrowband filter, Analog Filter Design1. The filter is executed as 8-th Butterworth filter. The signal from the filter exit enters the multiplier Product1, where the modulated signal is multiplied into sinusoidal harmonics with a frequency being equal to the frequency of the carrier signal. After multiplication the signal enters the analog low frequency filter (Analog Filter Design block), which is also executed as an 8-th Butterworth filter. This filter plays the role of a system integrator. Then, the signal from the Analog Filter Design exit is standardized using a procedure performed by Abs, Substract, and Product 2 and Divide blocks and analyzed.

For simulation of the communication line models without information coding, with Hamming code, Reed-Solomon codes, and $\mathrm{BCH}$ codes are designed. As an execution example, Figure 4 shows models performing data transmission without coding and with Hamming coding. The models include AWGN Channels with the possibility to vary line parameters such as SNR in dB and signal source capacity. Both models contain BPSK modulators and demodulators, as well as means for monitoring of data transmitted.

\section{SIMULATION RESULTS}

\subsection{Transmission of the Modulated Signal in Power Cables}

Simulation results for communication line models according Figure 3 are shown on Figure 5 as a Bode magnitude diagram (BMD) and a phase-frequency characteristic diagram (PFC). As may be inferred from the simulation results, BMD and PFC spectra coincide with results obtained by us formerly in MicroCap environment [1] almost completely. Taking into consideration that simulation principles in MicroCap and MATLAB environments are different, it is appropriate to assess the reliability of the obtained results as sufficiently high. It may be also insisted that the obtained results describe a real communication line designed for DHTS adequately.

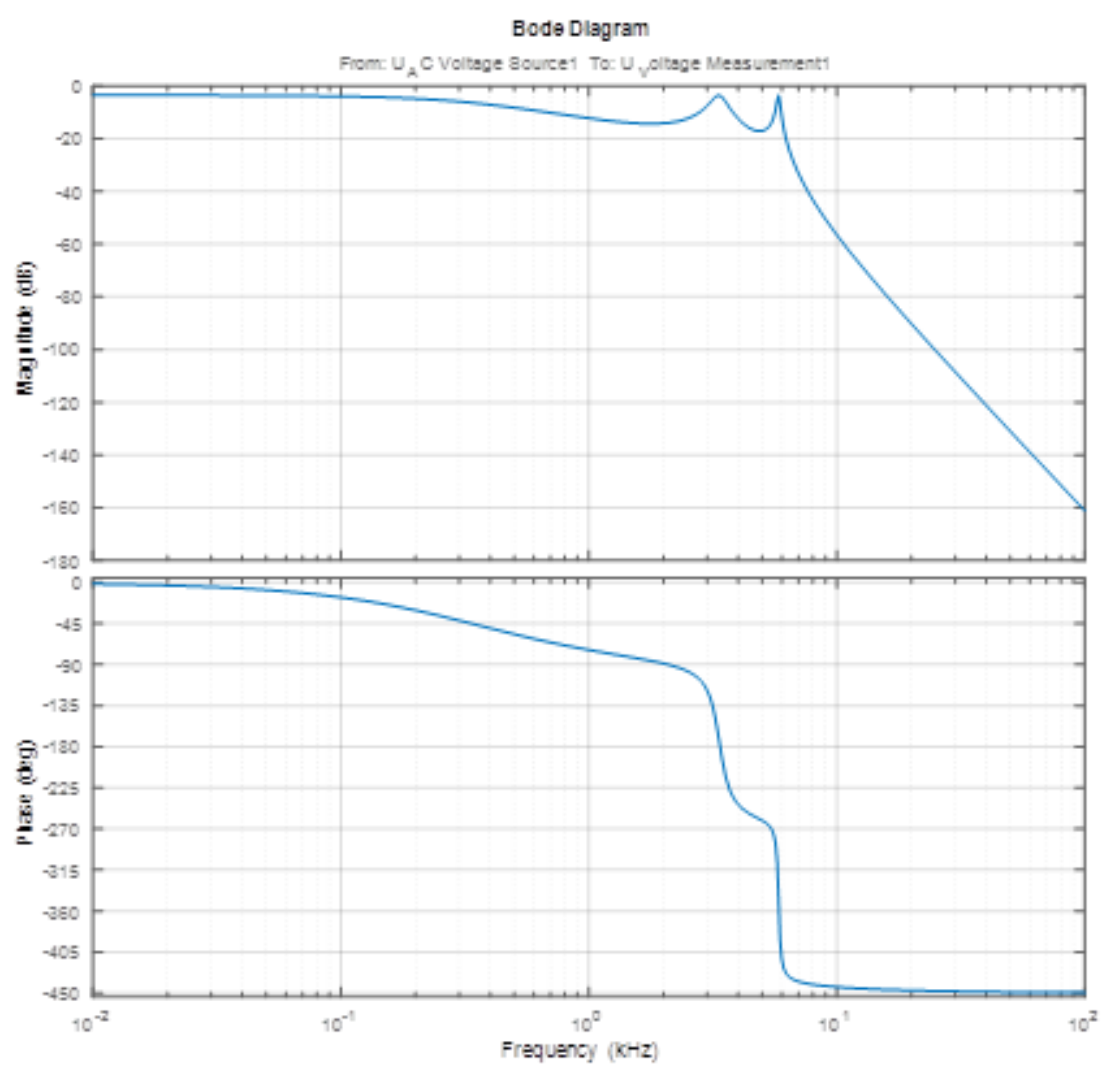

Figure 5. Communication line simulation results in MATLAB. The diagrams represent Bode magnitude (upper) and phase-frequency characteristics (lower) 
Further, origin of the carrier in the communication line is analyzed. The carrier amplitude for an incoming signal was set as approximately $30 \mathrm{~V}$ with the frequency of $6 \mathrm{kHz}$; whereas, the amplitude of the output signal was approximately $650 \mathrm{mV}$. In case of relatively good filtration and signal amplification there can be obtained an acceptable signal-noise ratio.

The next simulation stage is simulation of the bit stream transfer through the communication line using BPSK modulation. In case of BPSK modulation bit signals 0 and 1 are represented by $180^{\circ}$ phase shifts. For simulation the amplitude of the input harmonics was set as $30 \mathrm{~V}$, the frequency of the carrier signal was set as $6.2 \mathrm{kHz}$. After the modulated BPSK signal has passed through the communication line, it enters into the correlative demodulator (Figure 3). The route of the information signal in the communication line is shown on Fig. 6 in a form of oscilloscope records obtained in Scope1 block. Following signals can be seen on these oscilloscope records: input bit sequence (Figure 6a), bit sequence at demodulator's output (Figure 6b), signal on the communication line output and demodulator input (Figure 6c), signal after filtration by the narrowband filter Analog Filter Design1 (blue, Figure 6d), and signal at the output of low-frequency filter Analog Filter Design (red, Figure 6d). It should be noted that, due to the presence of integrating elements in the signal path (communication line, low-frequency filter), as well as specifics in demodulator operation, the output signal is slightly delayed, but this delay has no effect on data receiving correctness. Availability of a transition process at the start of simulation is also observed. Transition process availability can result in errors in some bits of data transmitted.

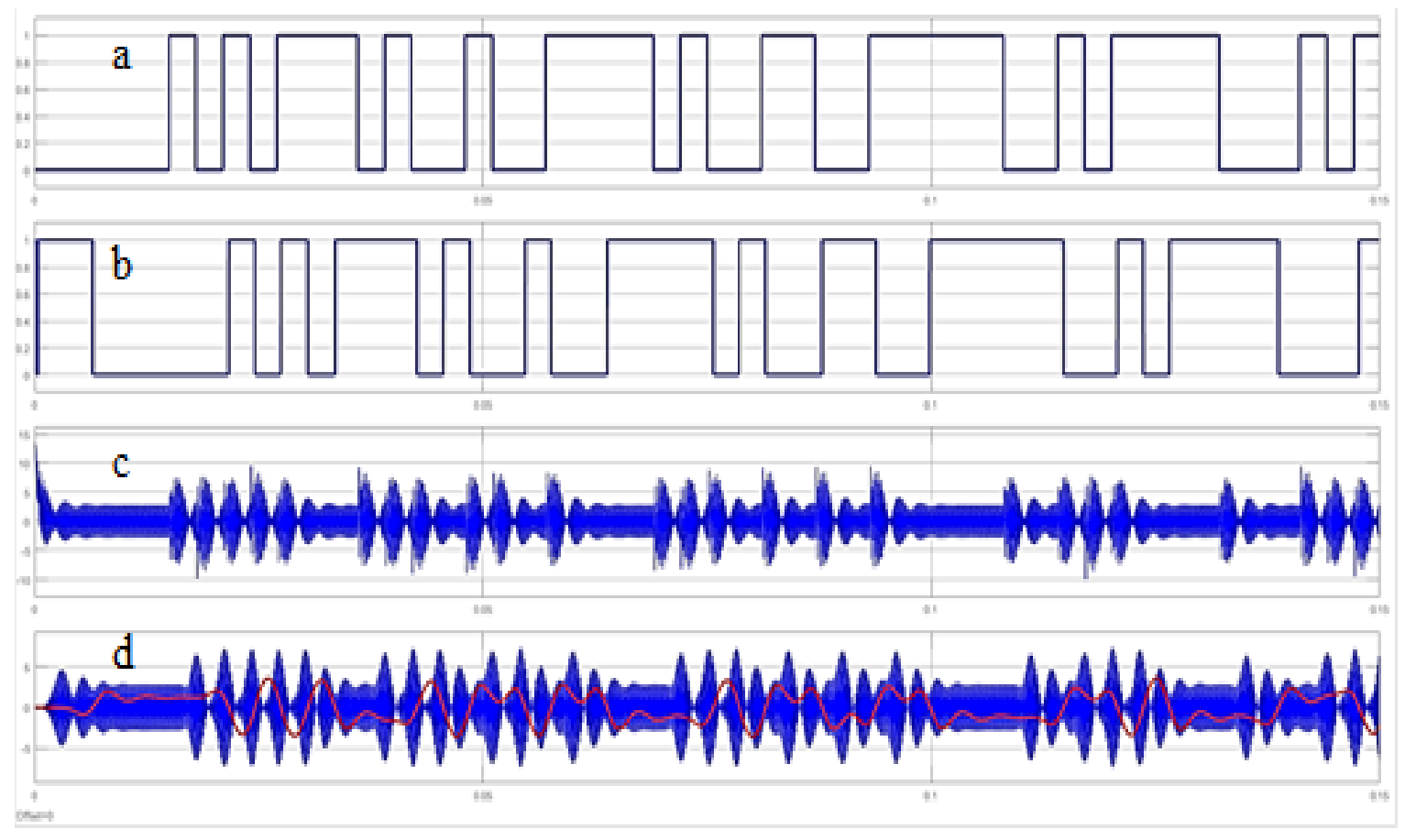

Figure 6. Oscilloscope records clarifying the signal path in the DHTS communication line model. (a) input bit sequence (b) bit sequence at demodulator's output (c) signal on the communication line output and demodulator input (d) signal after filtration by the narrowband filter Analog Filter Design1 (blue) and signal at the output of low-frequency filter Analog Filter Design (red)

Further, an emergency situation was simulated, when a phase fault/imbalance occurs. In other words, an uncompensated alternating SEM power supply voltage occurs in a neutral point in SEM. Simulation results are shown as oscilloscope records on Figure 7. Upon simulation results it is seen that, in case of a harmonic interference with the frequency of $50 \mathrm{kHz}$ and amplitude of approximately $500 \mathrm{~V}$, a $180^{\circ}$ phase shift of the modulated signal occurs at the moment of interference commutation. However, taking in consideration the phase shift at the moment of the interference commutation, the signal information element is transferred correctly, if the "phase-shift due to interference" is detected. If time moments when the phase shift due to interference occurs are not detected, group errors in data received there may arise. It may be the 
case that use of a quadrature modulator will be helpful in avoiding such problems in data transmission using BPSK modulation methods. Other drastic solution of this problem is use of BFSK modulation methods where such problems with signal phase-shifts do not arise.

As one can see from the simulation results, if the signal phase-shift is omitted, for example, in case of BFSK modulation, the harmonic interferences cause no damage to the data transmitted. The most efficient method for protection against harmonic interferences in DHTS communication lines is filtration. In this regard, the AWGN channel where all the interferences are only "white noise" was selected for further simulation of signal data transmission using different error-correcting codes.

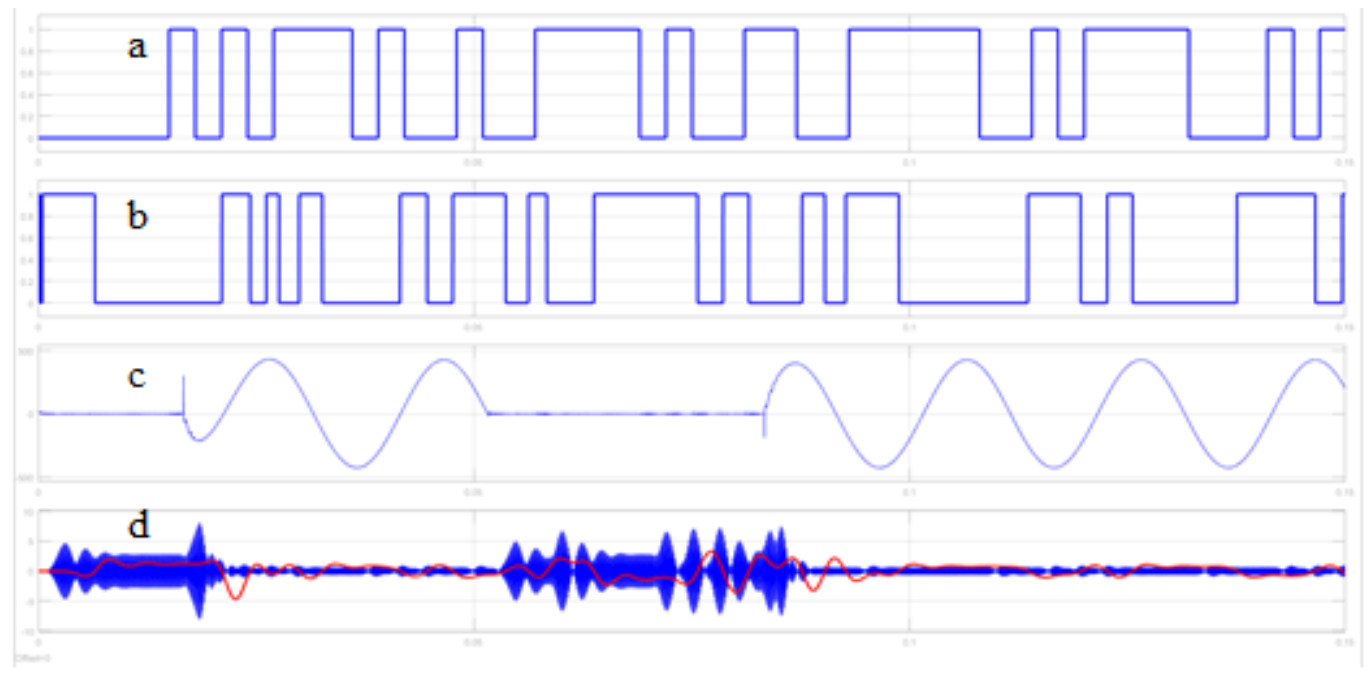

Figure 7. Oscilloscope records clarifying the signal path in the DHTS communication line model under the influence of an interference signal (a) input bit sequence (b) bit sequence at demodulator's output (c) signal with interference on the communication line output and demodulator input (d) signal after filtration by the narrowband filter Analog Filter Design1 (blue) and signal at the output of low-frequency filter Analog Filter Design (red)

In addition, a differential bit-phase manipulation (DBPSK) can be used for protection against "phase-shift due to interference" effects. The distinction of the DBPSK modulation method from BPSK consists in that special monitoring systems for signal demodulation shall be used in case of data transmission by the BPSK modulation method. Such monitoring systems (incoherent receiving devices) do not match with the phase of the carrier signal on the transmitter side and, therefore, cannot detect a random phase shift. Differential encoders and decoders are used for elimination of incoherent receiving problems. These encoder and decoder are installed before and after BPSK modulator and demodulator, respectively. However, experience has proven that the noise immunity of DBPSK method is less than one of BPSK method due to error multiplication at the decoding stage.

\subsection{Specific Details of Information Signal Transmission in Power Lines}

As can be seen from simulation results, information coding enables to weaken requirements on SNR for data transmission channels by $6 \mathrm{~dB}$ at the average. Simulation results are given in Table 1 . Two versions of data transmission were simulated with SNRs $5 \mathrm{~dB}$ and $1 \mathrm{~dB}$. As the simulation results has proven, in case of SNR $=5 \mathrm{~dB}$ Reed-Solomon codes and BCH codes enable to correct the errors occurred in full. However, the size of data transmitted increases by 7 and 3 times, respectively. In case of transmission of small data arrays such data size increase is acceptable. 
Table 1. Simulation Results for Data Transmission in DHTS Communication Lines with Different Coding Methods

\begin{tabular}{ccccc}
\hline \multirow{2}{*}{ Parameter } & \multicolumn{4}{c}{ Coding methods } \\
\cline { 2 - 4 } & W/o coding & Hamming code & Reed-Solomon code & BCH code \\
\hline $\begin{array}{c}\text { Number of bits } \\
\text { transmitted in the } \\
\text { line }\end{array}$ & 10000 & 17500 & 70000 & 30000 \\
$\begin{array}{c}\text { Number of } \\
\text { information bits }\end{array}$ & 10000 & & & 10000 \\
transmitted & & 10000 & 10000 & $\mathbf{5}$ \\
$\quad$ SNR, dB & $\mathbf{5}$ & $\mathbf{5}$ & $\mathbf{5}$ & 0 \\
Number of errors & 67 & 4 & 0 & $\mathbf{1}$ \\
Bit error rate & 0,0067 & 0,0004 & 0 & 32 \\
SNR, dB & $\mathbf{1}$ & $\mathbf{1}$ & $\mathbf{1}$ & 0,0032 \\
Number of errors & 585 & 235 & 40 & 0,004 \\
Bit error rate in & 0,0585 & 0,00235 & & 0 \\
data received & & & & \\
\hline
\end{tabular}

Simulation of data transmission through an AWGN-channel (Figure 4) with SNR $=1 \mathrm{~dB}$ has shown that the error probability increases by an order of magnitude more (see Table 1). Thereby, block codes (ReedSolomon and $\mathrm{BCH}$ ) enable to correct errors occurred almost in full. It is also seen from Table 1 that the simpler Hamming code can also be used successively, but only in availability of an appropriate data transmission channel, i.e. a channel with a high enough SNR.

Further, types of data transmission in DHTS communication lens with different coding methods were compared; in particular, a dependence of the bit error rate (BER) on transmission channel performance was simulated. Simulation results are shown on Figure 8.
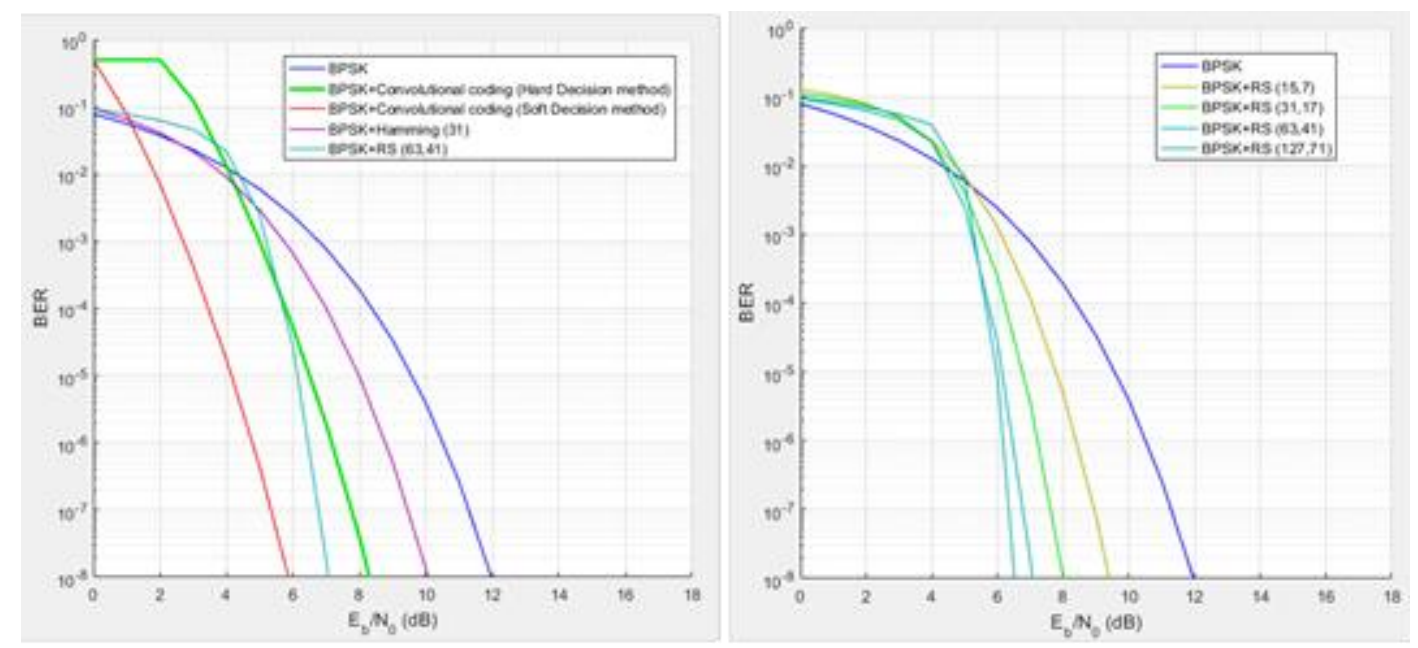

Figure 8. Simulation results for data transmission in DHTS communication lines using different signal coding methods (left) and Reed-Solomon code with different proportion of blocks in code word (right)

Simulation results are obtained for the case of data transmission using convolutional codes, hamming code, and Reed-Solomon code. Reed-Solomon code and Hamming code are presented with an optimum (in view of noise immunity) ratio of information bits to the total number of bits per word (given in brackets on the figure). Convolutional codes are presented with two types of the information decoding process (hard decision method and soft decision method). It can be seen that the best values of noise immunity are obtained for Reed-Solomon code and convolutional code with the soft decision method.

The dependence of the bit error rate on transmission channel quality was also simulated using ReedSolomon codes with different proportion of blocks in code word (Figure 8). It can be seen that the lager the size of the code word is, the more significantly the transmission reliability increases. Thus, it can be inferred from the plot that the optimum size of the code word for data transmission in communication lines is (63, $41),(127,71)$ and more. However the further increase of the code word size results in no significant increase of transmission reliability. 


\section{CONCLUSION}

Data transmission in a DHTS communication line presented as a power cable for SEM power supply has been simulated. Upon simulation results it has been found that the optimum signal modulation method for bit data transmission through power cables under conditions of severe interference harmonics and significant noise are phase modulation methods such as BPSK and DPBSK and frequency modulation methods such as BFSK and CPFSK. It has been also detected that using of error-correcting coding methods such as convolutional coding, Reed-Solomon codes, $\mathrm{BCH}$ coding enables to weaken requirements for data transmission channels and to increase information transmission reliability. Use of simple coding methods (Hamming codes) gives no significant improvements in data receiving quality and is efficient only in availability of an appropriate transmission channel.

\section{ACKNOWLEDGEMENTS}

Work on PNIER RFMEFI57815X0134 was performed with the financial support of the Ministry of Education and Science of the Russian Federation in the framework of the agreement №14.578.21.0134 on 27 October 2015.

\section{REFERENCES}

[1] E. J. Antheron, "Apparatus for transmitting instrumentation signals over power conductors," U.S. Patent, no. $5539375,1996$.

[2] O. Ghoreishi, et al., "Phase-to-Phase Communication Scheme for Downhole Monitoring Tool Design in Electrical Submersible Pump Systems," IEEE Transactions on Industry Applications, vol. 52(3), pp. 2077-2087, 2016.

[3] Th. Tran, et al., "High-Bitrate Downhole Telemetry System," in Proceedings of IEEE International Symposium on Power Line Communications and Its Applications (ISPLC-2015), 2015, pp. 280-284.

[4] J. Bailey, et al., "Data telemetry system for multi-conductor wirelines," U.S. Patent, no. US20030011490 A1, 2003.

[5] K. O. Khokhlov, et al., "Electric power converter with a wide input voltage range," International Journal of Power Electronics and Drive System (IJPEDS), vol. 7, is. 4, pp. 1269-1275, 2016.

[6] E. S. Trofimova, et al., "Downhole telemetry system reliability calculation and improvement" AIP Conference Proceedings, vol. 1886, no. 020028, 2017.

[7] A. V. Ishchenko, et al., "Simulation of communication line for down hole telemetry systems," AIP Conference Proceedings, vol. 1767, no. 020040, 2016.

[8] O. O. Rubtsova, et al., "Submersible telemetry system downhole unit model for the petroleum industry," AIP Conference Proceedings, vol. 1886, no 020055, 2017.

[9] Y. Bensalem, et al., "Modeling and Simulation of Induction Motor based on Finite Element Analysis," International Journal of Power Electronics and Drive System (IJPEDS), vol. 7, no. 4, pp. 1100-1109, 2016.

[10] M. Jannati, et al., "Modelling of a 3-Phase Induction Motor under Open-Phase Fault Using Matlab/Simulink," International Journal of Power Electronics and Drive System (IJPEDS), vol. 7, no. 4, pp. 1146-1152, 2016.

[11] Md R. Amin, et al., "Modelling of Virtual Synchronous Converter for Grid-Inverter Synchronization in Microgrids Applications," International Journal of Power Electronics and Drive System (IJPEDS), vol. 7, no. 4, pp. 1377$1385,2016$.

[12] I. A. Kalistratov, et al., "Simulation model of multi-antenna communication system with the space-time block coding," Modern scientific research and innovations, No. 6, pp. 4-10, 2013. 